Published in final edited form as:

Trends Biotechnol. 2014 April ; 32(4): 186-197. doi:10.1016/j.tibtech.2014.02.003.

\title{
Chemically programmed antibodies
}

\author{
Christoph Rader ${ }^{1,2}$ \\ ${ }^{1}$ Department of Cancer Biology, The Scripps Research Institute, Scripps Florida, 130 Scripps \\ Way \#2C1, Jupiter, FL 33458, USA. \\ ${ }^{2}$ Department of Molecular Therapeutics, The Scripps Research Institute, Scripps Florida, 130 \\ Scripps Way \#2C1, Jupiter, FL 33458, USA.
}

\begin{abstract}
Due to their unlimited chemical diversity, small molecules can rival monoclonal antibodies (mAbs) with respect to specificity and affinity for target molecules. However, key pharmacological properties of mAbs remain unmatched by small molecules. Chemical programming strategies have been developed for site-specific and covalent conjugation of small molecules to mAbs with unique reactivity centers. In addition to blending favorable features of small molecules and mAbs, chemically programmed antibodies (cpAbs) are economically attractive because they utilize the same mAb for a virtually unlimited number of target molecule specificities, reducing manufacturing costs and shortening drug discovery and development time. Preclinical studies and clinical trials have begun to demonstrate the broad utility of cpAbs for the treatment and prevention of human diseases.
\end{abstract}

\section{Keywords}

Pharmaceutical; monoclonal antibody; small molecule; site-specific conjugation; covalent conjugation

\section{Monoclonal an tibodies versus small molecules}

Monoclonal antibodies (mAbs) have become clinically and commercially highly successful pharmaceuticals. Over the past two decades $>30 \mathrm{mAbs}$ were approved by regulatory agencies in the USA and EU [1]. A similar number of mAbs is currently in phase III clinical trials [2]. mAbs have already benefitted millions of patients worldwide. Whereas cancer and autoimmune diseases have remained the dominant indications, mAbs for the treatment or prevention of infectious, cardiovascular, neurodegenerative, and other human diseases have also been marketed or have advanced to late stages of the drug pipeline. In some indications, such as CD20+ B-cell non-Hodgkin lymphoma (rituximab) and HER2+ breast cancer (trastuzumab), mAbs are now an integral part of standard first-line therapy. For certain rare human diseases, such as paroxysmal nocturnal hemoglobinuria (eculizumab), mAbs are or are projected to become the only approved drugs. In accord with their clinical success, global sales of mAbs totaled $>\$ 50$ billion in 2012 and are expected to rise at an annual growth rate of $>5 \%$ over the next decade (www.bccresearch.com).

\footnotetext{
(C) 2014 Elsevier Ltd. All rights reserved.

Corresponding author: Rader, C. (crader@scripps.edu).
}

Publisher's Disclaimer: This is a PDF file of an unedited manuscript that has been accepted for publication. As a service to our customers we are providing this early version of the manuscript. The manuscript will undergo copyediting, typesetting, and review of the resulting proof before it is published in its final citable form. Please note that during the production process errors may be discovered which could affect the content, and all legal disclaimers that apply to the journal pertain. 
What are the reasons behind the boom of mAbs? Key features at the center of both their clinical and commercial success are precision and pharmacology. The precision of mAbs is in one part founded on their high specificity and affinity for antigens and in another part due to the local confinement of the large antibody molecule $(\sim 150 \mathrm{kDa})$ to the circulatory system and interstitial space. Small molecules $(<1 \mathrm{kDa})$, by contrast, have unlimited access to nearly all extracellular and intracellular niches, making activity and toxicity profiles less predictable. The larger size of mAbs, along with its recycling through the neonatal Fc receptor $(\mathrm{FcRn})$ of endothelial cells, monocytes, and macrophages also results in a prolonged circulatory half-life when compared to small molecules. In addition to interacting with FcRn, the Fc fragment of mAbs in Immunoglobulin $\mathrm{G}(\mathrm{IgG})$ format, mediates their effector functions, including complement-dependent cytotoxicity (CDC) by recruiting complement protein $\mathrm{C} 1 \mathrm{q}$ and antibody-dependent cellular cytotoxicity (ADCC) and antibody-dependent cellular phagocytosis (ADCP) by interacting with $\mathrm{Fc} \gamma$ receptors expressed on NK cells, macrophages, and other leukocytes. CDC, ADCC, and ADCP are collectively known as the effector functions of mAbs. Fc optimization through rational design and directed evolution has allowed the tuning of the circulatory half-life as well as effector functions [3]. Thus, the Fc fragment is crucial for the favorable and tunable pharmacokinetic (PK) and pharmacodynamic (PD) properties of mAbs when compared to small molecules. Being small and synthetic, however, also has advantages. In contrast to mAbs, small molecules can readily diffuse through tissues and cells and can be made orally available. Small molecules are also cheaper to manufacture. The production of mAbs, which are heterogeneous glycoproteins expressed in mammalian cells and purified by a multistep chromatography process [4], is considerably more complex than the production of small molecules. Although the higher manufacturing costs contribute only a fraction to the current market price of brand name mAbs, they will limit the savings one can expect from generic and biosimilar $\mathrm{mAbs}$ in the future.

Chemically programmed antibodies (cpAbs) address some of these challenges by blending favorable features of mAbs and small molecules (Table 1, Figure 1). Pharmacologically, cpAbs equip small molecules with the PK and PD properties of the antibody molecule. Economically, cpAbs are expected to reduce manufacturing costs and shorten drug discovery and development time (Figure 2). This review delineates the molecular concept and architecture of cpAbs, discusses their preclinical and clinical performance, and delivers an outlook on this new class of pharmaceuticals.

\section{Molecular concept and architecture of cpAbs}

Conventional mAbs (Figure 1A) bind antigens by virtue of their complementaritydetermining regions (CDRs), which are on certain $\beta$-turns of the antiparallel $\beta$-sandwich that makes up the immunoglobulin (Ig) fold. Up to six CDRs, three from each light and heavy chain variable domain, can contribute to the antigen binding site, potentially providing large surface areas mediating the interaction of paratope (antibody) and epitope (antigen) [5]. The proportion and complementarity of this interface explains the high specificity and affinity with which antibodies can bind antigens. Antigen recognition by cpAbs is fundamentally different in that it is mediated by a synthetic component, which is site-specifically and covalently attached to an antibody component (Figure 1B).

The antibody component equips the synthetic component with the circulatory half-life, bivalence, and effector functions of conventional mAbs. Although not directly contributing to antigen recognition, the antibody component also endows the synthetic component with bulkiness, which can augment its ability to interfere with ligand-receptor interactions. The only formal requirement for the antibody component of cpAbs is a unique reactivity center. At a minimum, the antibody component is an $\mathrm{Fc}$ fragment with a unique reactivity center at 
the $N$ - or $C$-terminus. The synthetic component of cpAbs consists of: (i) a pharmacophore (a peptide, a peptidomimetic, or other small molecule) that binds with high specificity and affinity to an extracellular (membrane-bound or secreted) antigen; (ii) a reactive (usually electrophilic) group that permits site-specific and covalent conjugation to a unique reactive (usually nucleophilic) residue of the antibody component; and (iii) a linker spacing pharmacophore and reactive group.

In the most basic molecular assembly, termed $F c$-based $c p A b$, the synthetic component replaces the Fab fragment of a conventional mAb (Figure 1B center). Alternatively, the antibody component is a conventional $\mathrm{mAb}$ with a unique reactivity center in its paratope suitable for chemical programming. The resulting cpAb (Figure 1B left), termed $I g G$-based $c p A b$, closely resembles a conventional $\mathrm{mAb}$ with respect to shape and size. This molecular assembly is found in the first cpAbs that were described a decade ago and entered clinical trials shortly after [6]. Overall, irrespective of these various molecular assemblies, the antibody component of cpAbs serves as a carrying moiety and the synthetic component serves as a targeting moiety. Recently, Fab-based cpAbs have been introduced, where the antibody component serves simultaneously as carrying and targeting moiety [7] (Figure 1B right). Thus, Fab-based cpAbs have two targeting moieties and constitute a new class of bispecific antibodies.

A key characteristic of cpAbs is the site-specific conjugation of the synthetic component to the antibody component. A defined molecular assembly mandates site-specific conjugation. Corresponding to conventional mAbs in IgG format, which comprise two Fab and one Fc fragment (Figure 1A), cpAbs combine a fixed number of targeting and carrying moieties in a demarcated arrangement (Figure 1B). By contrast, random conjugation of synthetic component and antibody component results in a mixture of conjugates, exhibiting a range of stoichiometries and batch-to-batch variability. In addition to causing product heterogeneity, random conjugation can impair the pharmacological properties of the antibody component [8]. Another key characteristic of cpAbs is the covalent conjugation of the synthetic component to the antibody component. Covalent conjugation can be reversible or irreversible.

\section{Features of the antibody component of cpAbs}

The site-specific and covalent conjugation of a synthetic component requires the presence of unique reactivity centers in the antibody component. Three unique reactivity centers have been predominantly used to generate cpAbs: a reactive lysine $(\mathrm{K})$ residue in the paratope for the assembly of IgG-based cpAbs, engineered $N$-terminal cysteine (C) or $C$-terminal selenocysteine (U) residues for Fc-based cpAbs, and an engineered $C$-terminal selenocysteine residue for Fab-based cpAbs (Figure 1C). The unique reactivity centers that are defined by these three different natural amino acids are discussed separately in the following. In addition, an $N$-terminal alanine-lysine-threonine (AKT) tripeptide [9], which constitutes a substrate for pyridoxal 5'-phosphate-mediated transamination, and the unnatural amino acid para-acetylphenylalanine (pAcPhe) $[10,11]$ were recently deployed as unique reactivity centers for Fc-based and Fab-based cpAbs, respectively (Table 2).

Reactive lysine-The original concept of cpAbs [6] was based on a group of mAbs that were induced by reactive immunization of mice and then cloned, expressed, and purified by hybridoma technology $[12,13]$. Reactive immunization can be defined as the induction of antibodies that covalently bind a reactive immunogen [14]. A 1,3-diketone hapten was used as a reactive immunogen for the induction of antibodies with a reactive lysine residue in the paratope (Figure 3A). The crystal structure of one of the anti-1,3-diketone hapten mAbs, $33 \mathrm{~F} 12$, revealed that the paratope is a hydrophobic cleft $>11 \AA$ deep with the reactive lysine 
residue, K99, at its base [15]. K99 of the heavy chain variable domain of mAb 33F12 and other anti-1,3-diketone hapten mAbs lies in close proximity to the third CDR and arose by somatic mutation $[13,16]$. The hydrophobic microenvironment disfavors the protonation of the $\varepsilon$-amino group of $\mathrm{K} 99$, thereby substantially reducing its $\mathrm{pKa}$ and allowing a reversible covalent interaction with the 1,3-diketone hapten to form an enaminone stabilized by imineenamine tautomerism (Figure 3A). The covalent conjugation was confirmed in a crystal structure of mAb 33F12 in complex with the 1,3-diketone hapten [17]. Collectively, K99 of anti-1,3-diketone hapten mAbs has become an extensively proven unique reactivity center in antibody components of cpAbs. Nonetheless, unique reactivity centers in the paratope that mediate site-specific and covalent conjugation are not limited to reactive lysine residues, providing additional opportunities for the generation of cpAbs [18-22]. For example, alternative unique reactivity centers include cysteines engineered by rational design [19] and tyrosines selected from antibody libraries by phage display [21].

$\mathbf{N}$-terminal cysteine-As discussed above, natural amino acids in the microenvironment of the paratope can have unique reactivity. Outside of a paratope or in its absence, the availability of unique reactivity centers in the antibody component is limited. An engineered $\mathrm{N}$-terminal cysteine residue, $\mathrm{C} 1$, in $\mathrm{Fc}$ fragments provides an example of a unique reactivity center used for the generation of cpAbs in the absence of a paratope [23]. Exploiting the concept of native chemical ligation [24], the naturally occurring 1,2-aminothiol group of C1 is reacted with a thioester derivative resulting in the formation of an amide bond (Figure 3B).

C-terminal selenocysteine-Another example of a unique reactivity center used for the generation of cpAbs in the absence of a paratope is an engineered $C$-terminal selenocysteine residue, $\mathrm{U} 234$, in $\mathrm{Fc}$ fragments [25]. Selenocysteine, also known as the $21^{\text {st }}$ natural amino acid, is inserted at the $C$-terminus by equipping an antibody expression cassette with a TGA codon followed by a hexa-histidine encoding sequence, a TAA stop codon, and a 3 ' untranslated region (UTR) with a selenocysteine incorporation sequence (SECIS) element [25]. In mammalian cells (in the presence of the SECIS element and supplemental $1 \mu \mathrm{M}$ sodium selenite $\left(\mathrm{Na}_{2} \mathrm{SeO}_{3}\right)$ in the cell culture medium), the TGA codon (UGA in the mRNA), which is normally a stop codon, instructs the incorporation of a selenocysteine residue during translation [26]. As selenocysteine insertion at the TGA codon competes with termination, this expression cassette yields both $\mathrm{Fc}$ with and without $\mathrm{U} 234$. By virtue of the hexa-histidine encoding sequence that follows the TGA codon, Fc-stop and Fc-U-H 6 proteins can be efficiently separated by immobilized metal affinity chromatography (IMAC) $[7,25,27]$. However, as termination dominates selenocysteine insertion, $\mathrm{Fc}-\mathrm{U}-\mathrm{H}_{6}$ is produced at approximately five-fold lower levels than $\mathrm{Fc}$-stop protein, and the majority of dimeric $\mathrm{Fc}$ $\mathrm{U}-\mathrm{H}_{6}$ protein is composed of a hinge- $\mathrm{C}_{\mathrm{H}} 2-\mathrm{C}_{\mathrm{H}} 3$-stop and a hinge- $\mathrm{C}_{\mathrm{H}} 2-\mathrm{C}_{\mathrm{H}} 3-\mathrm{U}-\mathrm{H}_{6}$ half, resulting in the display of only one unique reactivity center in an asymmetric $\mathrm{Fc}$ fragment (Figure 1C). Interestingly, without further loss in yield, a second unique reactivity center, $\mathrm{U} 240$, can be added by placing a tetra-glycine-serine $\left(\mathrm{G}_{4} \mathrm{~S}\right)$ encoding sequence followed by a second TGA codon between the first TGA codon and the hexa-histidine encoding sequence [28]. Due to the larger size of selenium (34 electrons) compared to sulfur (16 electrons), the selenol group of selenocysteine (pKa 5.2) is more nucleophilic than the thiol group of cysteine (pKa 8.3) and can be selectively and rapidly conjugated to maleimide (Figure 3C) and iodoacetamide derivatives of small molecules under mildly acidic ( $\mathrm{pH} 5.2$ ) and mildly reducing $(0.1 \mathrm{mM}$ dithiothreitol) conditions without modifying any other amino acid residues in the antibody molecule [25]. Although requiring complex translation machinery [29], all parts necessary for selenocysteine insertion preexist in mammalian cells and can be exploited for antibody engineering. Endogenous antibodies do not contain 
selenocysteine residues, providing an opportunity for a unique reactivity center in engineered antibodies.

In addition to the $\mathrm{Fc}$ format, $\mathrm{mAbs}$ in $\mathrm{IgG}, \mathrm{scFv}-\mathrm{Fc}$, and $\mathrm{Fab}$ format have been engineered with $C$-terminal selenocysteine residues $[7,27,28]$. mAbs with a $C$-terminal selenocysteine can also be expressed in bacteria as demonstrated for the scFv format [30]. Although using the same codon, TGA, the prokaryotic selenocysteine insertion machinery differs from its eukaryotic counterpart in several respects, including the location of its SECIS element immediately downstream of the TGA codon, which confines the location of the selenocysteine residue to the $C$-terminus if amino acid sequence mutations are to be avoided. By contrast, the location of its SECIS element in the 3' UTR theoretically allows placing the selenocysteine residue at various locations without compromising downstream amino acid sequences. Nonetheless, a $C$-terminal location (upstream of a $\mathrm{His}_{6}$ tag) ensures that even larger synthetic components in cpAbs do not interfere with $\mathrm{Fc}$ fragment interactions that govern circulatory half-life and effector functions. Regardless of whether utilizing prokaryotic or eukaryotic selenocysteine insertion machineries, strategies for increasing the ratio of selenocysteine insertion over termination $[31,32]$ may prove important for translating cpAbs with a selenocysteine interface from preclinical studies to clinical trials.

Other unique reactivity centers-Site-specific and covalent conjugation of a synthetic component to an antibody component is also relevant in the generation of antibody-drug conjugates (ADCs) [33]. In contrast to cpAbs, ADCs use the antibody component for mediating antigen binding and the synthetic component for mediating cytotoxicity. Nonetheless, defined molecular assemblies involving unique reactivity centers are relevant for both ADCs and cpAbs. In addition to natural amino acids, such as engineered cysteines [34], genetically encoded unnatural amino acids, such as pAcPhe [35], have been used as unique reactivity centers in ADCs. Inserting genetically encoded unnatural amino acids via termination codons (typically TAG) requires an exogenous translation machinery with an orthogonal tRNA/aminoacyl-tRNA synthetase pair [36]. This strategy allows for the insertion of nucleophilic or electrophilic unnatural amino acids, affording a variety of conjugation chemistries.

mAbs with unique reactivity centers can also be generated enzymatically through a genetically encoded, typically $C$-terminal peptide tag $[37,38]$. For example, formylglycinegenerating enzyme converts the cysteine residue (thiol group) in the consensus pentapeptide sequence CXPXR to a formylglycine residue (aldehyde group). The electrophilic aldehyde group is then reacted with small molecules derivatized with nucleophilic aminooxy or hydrazide groups [39]. Other enzymes that have been used for site-specific and covalent conjugation of a synthetic component to an antibody component include sortase [40] and transglutaminase [41]. For therapeutic applications that necessitate repeated dosing, traceless or near traceless strategies, which replace all or most of the peptide tag with the small molecule, are preferred because they minimize the potential of immunogenicity [42]. Collectively, the strategies described in this paragraph provide a rich assortment of alternative routes to antibody components with unique reactivity centers.

\section{Features of the synthetic component of cpAbs}

In order to serve as synthetic components for cpAbs, small molecules must bind these antigens with high specificity and affinity. Suitable pharmacophores for the synthetic component of cpAbs were created by rational design or selected from chemical libraries. The ability to use one-bead-one-compound (OBOC) and other chemical libraries for developing synthetic components is a key incentive for cpAbs because it may allow 
identifying pharmacophores for targeting extracellular antigens with unknown high affinity binding sites for small molecules [43,44]. In addition, the selection of peptide libraries by phage display has yielded pharmacophores for synthetic components of cpAbs [45]. In fact, peptides derivatized with suitable linkers and reactive groups are used as synthetic components of first-generation cpAbs that have reached clinical trials (Table 2). An advantage these assemblies have over peptibodies [46], where a peptide is genetically fused to a $\mathrm{Fc}$ fragment, is that the linkage is not confined to the $\mathrm{N}$ - or the $\mathrm{C}$-terminus of the peptide but can involve amino acid residues in the middle, affording antibody-peptide conjugates with superior pharmacological properties [47]. In addition, whereas peptibodies are limited to peptides that consist of L-amino acids, cpAbs can also accommodate peptides with Damino acids, which have demonstrated stronger resistance to proteolytic degradation.

IgG-based and symmetric Fc-based cpAbs dimerize the pharmacophore by virtue of their two unique reactivity centers (Figure 1B). The ability to bind extracellular antigens bivalently rather than monovalently can offset shortcomings of pharmacophores that bind their target molecules with suprananomolar to submicromolar affinity. An avidity gain of asymmetric Fc-based cpAbs and Fab-based cpAbs with one unique reactivity center can be achieved by synthesizing synthetic components with homodimeric pharmacophores [7,48]. Notably, neither approach substantially increases the size of cpAbs. In addition to homodimeric pharmacophores, synthetic components with heterodimeric pharmacophores engaging two different extracellular antigens have been deployed to afford bispecific cpAbs as discussed below. It is conceivable that synthetic components of cpAbs could incorporate additional moieties that augment the pharmacological properties of cpAbs. These may include cytotoxic drugs or radioisotopes for indications that require efficient target cell destruction or peptides, peptidomimetics, or other small molecules that extend the circulatory half-life of cpAbs by binding to serum proteins. For certain applications it may be advantageous to add these moieties to the already assembled cpAb by click chemistry [49]. In addition to small synthetic molecules, the synthetic component in cpAbs can comprise large synthetic molecules, such as RNA or DNA aptamers [9,50] (Table 2), which can be selected for high specificity and affinity from oligonucleotide libraries. Collectively, the ability to tailor specificity, valence, potency, and circulatory half-life through chemical synthesis is a distinctive asset of cpAbs.

\section{Bispecific cpAbs}

Bispecific antibodies are a class of next-generation antibody pharmaceuticals enabling simultaneous engagement of two antigens or epitopes. Bispecific binding can augment specificity or potency or both. For example, through acquiring bispecificity, mAbs can be made more specific for target cells by requiring the presence of two different cell surface antigens and can be made more potent by simultaneously antagonizing two different signaling pathways [51]. Furthermore, bispecific antibodies can exert cytotoxicity by recruiting and activating endogenous immune cells [51]. This is achieved by combining specificities for target and effector cells in one molecule. Bispecific cpAbs, in which one or both specificities are mediated by a synthetic component, have also been described $[7,10,11,48,52]$. There are many possible architectures for bispecific cpAbs (Figure 4). A first group among these utilizes two different monospecific synthetic components. These are either conjugated to two identical unique reactivity centers (resulting in mixtures of bivalent monospecific and monovalent bispecific cpAbs) or to two orthogonal unique reactivity centers. For example, an antibody component that pairs a reactive lysine residue in the paratope with a $C$-terminal selenocysteine residue affords two orthogonal unique reactivity centers that can be simultaneously or sequentially conjugated to two different synthetic components. A second group of bispecific cpAbs utilizes a bispecific synthetic component. Published examples include trifunctional synthetic components that combine specificities for 
two different extracellular antigens with a reactive group [48,52]. A third group of bispecific cpAbs make use of antibody components with a paratope that remains untouched by chemical programming. Conjugating a monospecific synthetic component to a unique reactivity center in these antibody components affords two antigen binding sites, one provided by the antibody component and one provided by the synthetic component. With a molecular weight of $50 \mathrm{kDa}$, Fab-based bispecific cpAbs [7,10,11] share the same size with a newer class of conventional bispecific antibodies that brings target and effector cells into close proximity to enable cytolytic synapses and avoids systemic effector cell activation through monovalent rather than bivalent engagement in the absence of target cells [53,54]. In addition to the above discussed economic and pharmacological advantages cpAbs have over mAbs (Table 1), bispecific cpAbs may be more adept at reaching concealed epitopes in membrane proximal locations for the formation of cytolytic synapses.

\section{Comparison of cpAbs with ARMs}

Chemical programming of a unique reactivity center in the paratope of an $\operatorname{IgG}$ can be achieved by either covalent or non-covalent conjugation. In the latter case, the reactive moiety in the synthetic component is replaced with a hapten that binds with high affinity to the paratope. For example, fluorescein can serve as a hapten for the non-covalent conjugation of a synthetic component to an anti-fluorescein mAb. Notably, non-covalent conjugations enable the chemical programming of endogenous antibodies. In this concept [55], which has been reviewed elsewhere [56], the synthetic component serves as an antibody-recruiting molecule (ARM). ARMs can recruit preexisting endogenous antibodies that recognize certain haptens, such as galactosyl-a-(1,3)-galactose (a-Gal) or 2,4dinitrophenol (DNP). Alternatively, ARMs can recruit endogenous antibodies that were triggered by immunization with hapten-carrier conjugates. For example, in a phase I clinical trial [57], patients with metastatic renal cell carcinoma were first vaccinated with fluorescein isothiocyanate (FITC; hapten) conjugated to keyhole limpet hemocyanin (KLH; carrier) and were then treated with a folate-FITC ARM. Folate (vitamin B9) binds with high affinity to folate receptor 1 (FOLR1), which is expressed on the cell surface of tumor cells. Thus, the folate-FITC ARM decorates tumor cells and recruits endogenous anti-FITC antibodies that kill the tumor cells by mediating CDC, ADCC, and ADCP. An advantage that ARMs have over cpAbs is that they can be made orally available. Nonetheless, compared to ARMs that assemble with endogenous antibodies of varying quantity and quality in vivo, preassembled cpAbs afford pharmacological control over the antibody component. In case of IgG-based cpAbs, which can be preassembled covalently or non-covalently, covalent conjugation of the synthetic component has the advantage that the resulting cpAb is a single molecule rather than a mixture of two molecules, which generally expedites the approval process of Investigational New Drug (IND) applications by regulatory agencies prior to commencing clinical trials.

\section{Applications of cpAbs}

\section{Targeting integrins}

Several integrins, which are expressed on tumor cells and tumor endothelial cells, have high affinity binding sites for tripeptide motifs, such as RGD (integrin av $\beta 3$, av $\beta 5$, av $\beta 6$, and a5 $\beta 1$ ) and LDV (integrins $\alpha 4 \beta 1$ and $\alpha 4 \beta 7$ ). These tripeptide motifs are present in extracellular matrix and cell surface proteins that bind to integrins. As linear or cyclic peptides, they potently antagonize these interactions and induce apoptosis. To improve the pharmacological properties of small molecules that target and antagonize integrins, a large variety of peptidomimetics have been developed in academia and industry [58]. Tapping into this resource of extensive chemical diversity, the concept of cpAbs was established with synthetic components having an integrin-targeting and antagonizing peptidomimetic as 
pharmacophore (Table 2). The pharmacophore used in the first cpAbs was developed by rational design as orally available RGD peptidomimetic that antagonizes integrins av $\beta 3$ and $\operatorname{av\beta 5}[59]$.

In the first reports of cpAbs, the anti-1,3-diketone hapten mAb 38C2 [12,15], and subsequently its humanized version $\mathrm{mAb}$ h38C2 [60], were conjugated to 1,3-diketone derivatives of proapoptotic peptidomimetics that bind with high specificity and affinity to integrins $\alpha_{v} \beta_{3}$ and $\alpha_{v} \beta_{5}[6,61]$. Chemical programming endowed $m A b 38 C 2$ with the ability to bind and kill tumor cells and tumor endothelial cells expressing these integrins. In mouse models, the cpAb prolonged the circulatory half-life of the peptidomimetic $>100$-fold and increased its cytotoxicity >1000-fold, with effector functions, such as CDC and ADCC, playing contributing roles $[6,62]$.

Notably, in addition to the preassembled cpAb, which was generated in vitro by simply incubating $\mathrm{mAb} 38 \mathrm{C} 2$ with a twice equimolar concentration of the 1,3-diketone derivative for two hours at room temperature, antibody component (given intravenously) and synthetic component (given intraperitoneally) also spontaneously assembled in vivo [6]. Prompted by this finding, 1,3-diketone derivatives were also shown to serve as ARMs for endogenous antibodies triggered by immunization [63]. With the preassembled cpAb being a preferred IND entity, however, subsequent studies with mAbs 38C2 and h38C2 switched the electrophilic group of the synthetic component from 1,3-diketone to 2-azetidinone ( $\beta$ lactam), which affords irreversible covalent conjugation to K99 [64]. Irreversible covalent conjugation to $\mathrm{mAb} 38 \mathrm{C} 2$ and other aldolase mAbs was also achieved with a vinylketone released from its stable acetone aldol adduct by the catalytic activity of the reactive lysine residue [65]. Validating the notion of broad utility of a single antibody component, an increasing number of preclinical studies have used chemically programmed mAbs $38 \mathrm{C} 2$ and h38C2 to target a multitude of extracellular antigens involved in cancer and other human diseases (Table 2).

Fc fragments with an engineered $\mathrm{C} 1$ were expressed in yeast by inserting the cleavage site of an endogenous yeast protease, Kex2, immediately upstream of the cysteine residue and downstream of a yeast secretion signal peptide [23,66]. For proof of concept, $\mathrm{C} 1$ was then reacted with a thioester derivative of the cyclic RGDfK peptide [23]. The resulting cpAb was shown to target tumor cells expressing integrin $\alpha_{\mathrm{v}} \beta_{3}$. Antibody fragments with $N$ terminal cysteine residues have also been expressed in bacteria [67] and mammalian cells [68], and these were reacted with small molecules derivatized with an aldehyde group to yield thiazolidine heterocycles.

For initial proof of concept of Fc fragments with an engineered $C$-terminal selenocysteine, U234 was reacted with a maleimide derivative of the peptidomimetic LLP2A. LLP2A is an orally available LDV-mimicking peptidomimetic that had been selected from an OBOC chemical library for binding to integrin a $4 \beta 1$ with picomolar affinity [69]. Chemical programming endowed both antibody component and synthetic component with pharmacological advantages. Fc-U-H6 acquired the ability to target tumor cells expressing integrin $\alpha 4 \beta 1$ and to block the interaction of integrin $\alpha 4 \beta 1$ with vascular cell adhesion molecule 1 (VCAM1). LLP2A acquired a prolonged circulatory half-life and the ability to be delivered via the lung to the blood by FcRn-mediated transcytosis [25] (Table 1).

\section{Targeting other membrane-bound antigens}

In addition to integrins, a number of other cell surface receptors with high affinity binding sites for small molecules have been targeted by cpAbs (Table 2). These include receptors with natural small molecule ligands, such as FOLR1 [7,11], and receptors with natural peptide ligands, such as endothelin receptor type A [70], luteinizing hormone receptor [48] 
and kappa opioid receptor [71]. Other non-integrin cell surface receptors with high affinity binding sites for small molecules that were derived by rational design or selected from chemical libraries have also been described as targets of cpAbs. This list includes prostatespecific membrane antigen (PSMA) [11], CCR5 [72], gp120 of HIV-1 [73], and neuraminidase of the influenza virus [74]. In addition, protein tyrosine kinase-7 and cell surface IgM have been targeted with cpAbs that deploy DNA aptamers as synthetic component [9]. Furthermore, cpAbs have been used to extend the short circulatory half-lives of small therapeutic peptidomimetics, peptides, and proteins, including analogs of thrombospondin-1 [75,76], glucagon-like peptide-1 (GLP-1), human growth hormone [77], and FGF-21 [78].

Simultaneous targeting of two different cell surface receptors was demonstrated for bispecific cpAbs in Fab format that engage CD3 on T cells via their antibody component and integrin a $4 \beta 1$ [7], FOLR1 [7,11], or PSMA [10] on target cells via their synthetic component. Bispecific cpAbs can also engage two different cell surface receptors on the same target cell [48].

\section{Targeting secreted antigens}

An important application of cpAbs has been the neutralization of growth factors promoting tumor growth and angiogenesis, including angiopoietin-2 [47,52,79], VEGF [52], and placental growth factor-1 [45]. Simultaneous neutralization of two growth factors was demonstrated for a bispecific cpAb in IgG format. Specifically, the $\varepsilon$-amino group in a lysine residue of a peptide neutralizing angiopoietin-2 was linked to the $\varepsilon$-amino group in a lysine residue of a peptide neutralizing VEGF via a polyethylene glycol linker displaying a branched out 2-azetidinone for chemical programming of mAb h38C2 [52]. The resulting bispecific cpAb was shown to simultaneously bind angiopoietin-2 and VEGF and to potently inhibit tumor growth and angiogenesis in mouse xenograft models.

\section{Clinical trials with cpAbs}

The versatility of cpAbs, which is the result of generic molecular assembly of a variable synthetic component with an invariable antibody component (Figure 2), along with the ability to produce the antibody component by Good Manufacturing Practice (GMP) at kilogram scale, has facilitated the rapid expansion of the preclinical and clinical pipeline of cpAbs based on mAb h38C8, also known as CovX-Body platform. CovX, Inc. (San Diego, CA), which was acquired by Pfizer, Inc. (New York, NY) in 2008, advanced several CovXBodies to clinical trials. The antibody component, mAb h38C2 (CVX-2000), was expressed in mammalian cells and purified by sequential Protein A and ion exchange chromatography [52]. Four CovX-Bodies have been investigated in eight clinical trials registered at ClinicalTrials.gov (Table 2). As reported on abstracts published by the American Society of Clinical Oncology (ASCO), CVX-045, which mimics thrombospondin-1, CVX-060, which neutralizes angiopoietin-2, and CVX-241, which is a bispecific cpAb that neutralizes angiopoietin-2 and VEGF, were generally found to be well tolerated and to permit dosing once a week. Human anti-cpAb antibodies were detected at low titers in a portion of treated patients without clear effects on pharmacokinetics and safety. Following completion of the phase I clinical trial, CVX-060 was tried in combination with already approved multitargeting receptor tyrosine kinase inhibitors for the treatment of renal cell carcinoma. This includes an active multicenter phase I/II clinical trial (NCT00982657) for CVX-060 plus sunitinib and a terminated multicenter phase II clinical trial (NCT01441414) for CVX-060 plus axitinib. As reported on ClinicalTrials.gov, the latter was prematurely discontinued due to an unexpected frequency of arterial and venous thrombotic events. Also terminated was a phase I clinical trial with the bispecific cpAb CVX-241. Despite a very promising PK and PD profile in extensive preclinical studies [52], it was reported on ClinicalTrials.gov that the 
circulatory half-life of at least one of its two chemically programmed specificities was shorter than expected. There were no safety concerns with CVX-241. CovX-Bodies have also been developed for indications other than cancer. CVX-096, which mimics GLP-1, was evaluated in three phase I clinical trials for the treatment of type II diabetes mellitus, which collectively enrolled approximately 250 patients. No data showing the clinical safety and activity of CVX-096 has been published yet. CVX-096 was recently discontinued from the Pfizer pipeline along with CVX-045, CVX-060, and CXV-241. At this time it is not known when and if other CovX-Bodies with promising PK and PD profiles [45,71,78] will be translated from preclinical studies to clinical trials. Notably, all clinically investigated CovX-Body used peptides as synthetic components (Table 2), not taking full advantage of the unlimited chemical diversity of small molecules that can provide superior specificity, affinity, and stability. Medicinal chemistry approaches toward strengthening the PK and PD profiles of peptides [80] may also help improve the pharmacological performance of corresponding cpAbs.

\section{Concluding remarks}

A key aspect driving the preclinical and clinical development of cpAbs is their versatility. It only requires the cloning, expression, and purification of a single protein (i.e., the antibody component), which is then chemically programmed with small molecules (i.e., the synthetic components) for targeting a virtually unlimited number and variety of extracellular antigens. Both targeting and carrying moieties of cpAbs are experiencing rapid progress with respect to optimizing their pharmacological properties and broadening their scope for the treatment and prevention of human diseases. Several hundred patients treated with first-generation cpAbs constitute a robust platform for further advancing this new class of pharmaceuticals at the interface of chemistry and biology.

\section{Acknowledgments}

I thank Drs. Thomas Kodadek and Even Walseng for comments on the manuscript.

\section{Glossary}

\section{Antibody-dependent cellular cytotoxicity (ADCC)}

Antibody-dependent
cellular phagocytosis
(ADCP)

Bispecific antibody

$\mathbf{C}_{\mathrm{H}} \mathbf{1}$

$\mathrm{C}_{\mathrm{H}} 2$

$\mathrm{C}_{\mathrm{H}} 3$

$\mathrm{C}_{\mathrm{L}}$

Chemically programmed antibody (cpAb) lysis of an antibody-decorated target cell by an Fc $\gamma$ receptor-expressing effector cell, such as an NK cell

engulfment of an antibody-decorated target cell by an $\mathrm{Fc} \gamma$ receptor-expressing effector cell, such as a macrophage

engineered antibody that binds to two different epitopes monovalently or multivalently

first constant domain of the heavy chain

second constant domain of the heavy chain

third constant domain of the heavy chain

constant domain of the light chain

molecularly defined covalent composition of a variable synthetic component that serves as targeting moiety with an invariable antibody component that serves as carrying moiety 


\section{Complement-dependent cytotoxicity (CDC)}

\section{Complementarity- determining region (CDR)}

Epitope

Fab

Fc

Fc $\gamma$ receptor

Hapten

Immunoglobulin G (IgG)

Monoclonal antibody (mAb)

Neonatal Fc receptor

(FcRn)

Paratope

Peptidomimetic

Pharmacophore

Reactive immunization

Unique reactivity center

$\mathbf{V}_{\mathbf{H}}$

$\mathbf{V}_{\mathbf{L}}$ lysis of an antibody-decorated target cell by triggering the complement cascade

peptide loop in the $\mathrm{V}_{\mathrm{H}}$ or $\mathrm{V}_{\mathrm{L}}$ domain that is involved in antigen binding

antibody binding site of an antigen

antigen binding fragment of an antibody containing the $\mathrm{V}_{\mathrm{H}}$, $\mathrm{C}_{\mathrm{H}} 1, \mathrm{~V}_{\mathrm{L}}$, and $\mathrm{C}_{\mathrm{L}}$ domains

crystallizable fragment of an antibody containing two $\mathrm{C}_{\mathrm{H}} 2$ and two $\mathrm{C}_{\mathrm{H}} 3$ domains

cell surface receptor of IgG including CD16, CD32, and CD64

small molecule that elicits an immune response only when conjugated to a macromolecular carrier such as a protein

most common antibody isotype in the blood

natural or engineered antibody that binds one epitope monovalently or multivalently

cell surface receptor of IgG that mediates $\mathrm{IgG}$ transcytosis and salvage

antigen or hapten binding site of an antibody

small molecule that mimics a peptide

moiety of a small molecule that binds to a target molecule immunization with a hapten-carrier conjugate that elicits antibodies that covalently bind to the hapten

amino acid residue(s) in the antibody mediating sitespecific and covalent conjugation of a small molecule variable domain of the heavy chain variable domain of the light chain

\section{References}

1. Reichert JM. Marketed therapeutic antibodies compendium. mAbs. 2012; 4:413-415. [PubMed: 22531442]

2. Reichert JM. Antibodies to watch in 2013: Mid-year update. mAbs. 2013; 5:513-517. [PubMed: 23727858]

3. Strohl WR. Optimization of Fc-mediated effector functions of monoclonal antibodies. Curr Opin Biotechnol. 2009; 20:685-691. [PubMed: 19896358]

4. Kelley B. Industrialization of mAb production technology: the bioprocessing industry at a crossroads. mAbs. 2009; 1:443-452. [PubMed: 20065641]

5. Davies DR, Cohen GH. Interactions of protein antigens with antibodies. Proc Natl Acad Sci U S A. 1996; 93:7-12. [PubMed: 8552677]

6. Rader C, et al. Chemically programmed monoclonal antibodies for cancer therapy: adaptor immunotherapy based on a covalent antibody catalyst. Proc Natl Acad Sci U S A. 2003; 100:53965400. [PubMed: 12702756] 
7. Cui H, et al. Chemically programmed bispecific antibodies that recruit and activate T cells. J Biol Chem. 2012; 287:28206-28214. [PubMed: 22761439]

8. Boylan NJ, et al. Conjugation si te heterogeneity causes variable electrostatic properties in $\mathrm{Fc}$ conjugates. Bioconjug Chem. 2013; 24:1008-1016. [PubMed: 23777335]

9. Netirojjanakul C, et al. Synthetically modified Fc domains as building blocks for immunotherapy applications. Chemical Science. 2013; 4:266-272.

10. Kim CH, et al. Bispecific small molecule-antibody conjugate targeting prostate cancer. Proc Natl Acad Sci U S A. 2013; 110:17796-17801. [PubMed: 24127589]

11. Kularatne SA, et al. Recruiting cytotoxic T cells to folate-receptor-positive cancer cells. Angew. Chem. 2013; 125:12323-12326.

12. Wagner J, et al. Efficient aldolase catalytic antibodies that use the enamine mechanism of natural enzymes. Science. 1995; 270:1797-1800. [PubMed: 8525368]

13. Karlstrom A, et al. Using antibody catalysis to study the outcome of multiple evolutionary trials of a chemical task. Proc Natl Acad Sci U S A. 2000; 97:3878-3883. [PubMed: 10760259]

14. Tanaka F, Barbas CF 3rd. Reactive immunization: a unique approach to catalytic antibodies. J Immunol Methods. 2002; 269:67-79. [PubMed: 12379353]

15. Barbas CF 3rd, et al. Immune versus natural selection: antibody aldolases with enzymic rates but broader scope. Science. 1997; 278:2085-2092. [PubMed: 9405338]

16. Zhu X, et al. The origin of enantioselectivity in aldolase antibodies: crystal structure, site-directed mutagenesis, and computational analysis. J Mol Biol. 2004; 343:1269-1280. [PubMed: 15491612]

17. Zhu X, et al. Direct observation of an enamine intermediate in amine catalysis. J Am Chem Soc. 2009; 131:18206-18207. [PubMed: 19968282]

18. Wirsching P, et al. Reactive immunization. Science. 1995; 270:1775-1782. [PubMed: 8525366]

19. Chmura AJ, et al. Antibodies with infinite affinity. Proc Natl Acad Sci U S A. 2001; 98:84808484. [PubMed: 11447282]

20. Cesaro-Tadic S, et al. Turnover-based in vitro selection and evolution of biocatalysts from a fully synthetic antibody library. Nat Biotechnol. 2003; 21:679-685. [PubMed: 12754520]

21. Reshetnyak AV, et al. Routes to covalent catalysis by reactive selection for nascent protein nucleophiles. J Am Chem Soc. 2007; 129:16175-16182. [PubMed: 18044899]

22. Trisler K, et al. A metalloantibody that irreversibly binds a protein antigen. J Biol Chem. 2007; 282:26344-26353. [PubMed: 17617633]

23. Xiao J, et al. Targeting a homogeneously glycosylated antibody Fc to bind cancer cells using a synthetic receptor ligand. J Am Chem Soc. 2009; 131:13616-13618. [PubMed: 19728704]

24. Dawson PE, et al. Synthesis of proteins by native chemical ligation. Science. 1994; 266:776-779. [PubMed: 7973629]

25. Hofer T, et al. An engineered selenocysteine defines a unique class of antibody derivatives. Proc Natl Acad Sci U S A. 2008; 105:12451-12456. [PubMed: 18719095]

26. Hatfield DL, Gladyshev VN. How selenium has altered our understanding of the genetic code. Mol Cell Biol. 2002; 22:3565-3576. [PubMed: 11997494]

27. Hofer T, et al. Molecularly defined antibody conjugation through a selenocysteine interface. Biochemistry. 2009; 48:12047-12057. [PubMed: 19894757]

28. Li X, et al. Antibody conjugation via one and two C-terminal selenocysteines. Methods. 2014; 65:133-138. [PubMed: 23756202]

29. Lobanov AV, et al. Dual functions of codons in the genetic code. Crit Rev Biochem Mol Biol. 2010; 45:257-265. [PubMed: 20446809]

30. Johansson L, et al. Exploiting the 21st amino acid-purifying and labeling proteins by selenolate targeting. Nat Methods. 2004; 1:61-66. [PubMed: 15782154]

31. Thyer R, et al. Engineered rRNA enhances the efficiency of selenocysteine incorporation during translation. J Am Chem Soc. 2013; 135:2-5. [PubMed: 23256865]

32. Novoselov SV, et al. A highly efficient form of the selenocysteine insertion sequence element in protozoan parasites and its use in mammalian cells. Proc Natl Acad Sci U S A. 2007; 104:78577862. [PubMed: 17470795] 
33. Adair JR, et al. Antibody-drug conjugates - a perfect synergy. Expert Opin Biol Ther. 2012; 12:1191-1206. [PubMed: 22650648]

34. Junutula JR, et al. Site-specific conjugation of a cytotoxic drug to an antibody improves the therapeutic index. Nat Biotechnol. 2008; 26:925-932. [PubMed: 18641636]

35. Axup JY, et al. Synthesis of site-specific antibody-drug conjugates using unnatural amino acids. Proc Natl Acad Sci U S A. 2012; 109:16101-16106. [PubMed: 22988081]

36. $\mathrm{Kim} \mathrm{CH}$, et al. Protein conjugation with genetically encoded unnatural amino acids. Curr Opin Chem Biol. 2013; 17:412-419. [PubMed: 23664497]

37. Rashidian M, et al. Enzymatic Labeling of Proteins: Techniques and Approaches. Bioconjug Chem. 2013; 24:1277-1294.

38. Rabuka D. Chemoenzymatic methods for site-specific protein modification. Curr Opin Chem Biol. 2010; 14:790-796. [PubMed: 21030291]

39. Wu P, et al. Site-specific chemical modification of recombinant proteins produced in mammalian cells by using the genetically encoded aldehyde tag. Proc Natl Acad Sci U S A. 2009; 106:30003005. [PubMed: 19202059]

40. Swee LK, et al. Sortase-mediated modification of alphaDEC205 affords optimization of antigen presentation and immunization against a set of viral epitopes. Proc Natl Acad Sci U S A. 2013; 110:1428-1433. [PubMed: 23297227]

41. Strop P, et al. Location matters: site of conjugation modulates stability and pharmacokinetics of antibody drug conjugates. Chem Biol. 2013; 20:161-167. [PubMed: 23438745]

42. Bernardes GJ, et al. Site-specific chemical modification of antibody fragments using traceless cleavable linkers. Nat Protoc. 2013; 8:2079-2089. [PubMed: 24091555]

43. Kodadek T. Synthetic receptors with antibody-like binding affinities. Curr Opin Chem Biol. 2010; 14:713-720. [PubMed: 20674471]

44. Aina $\mathrm{OH}$, et al. From combinatorial chemistry to cancer-targeting peptides. Mol Pharm. 2007; 4:631-651. [PubMed: 17880166]

45. Bower KE, et al. Evolution of potent and stable placental-growth-factor-1-targeting CovX-bodies from phage display peptide discovery. J Med Chem. 2011; 54:1256-1265. [PubMed: 21280651]

46. Shimamoto G, et al. Peptibodies: A flexible alternative format to antibodies. mAbs. 2012; 4:586591. [PubMed: 22820181]

47. Huang H, et al. Specifically targeting angiopoietin-2 inhibits angiogenesis, Tie2-expressing monocyte infiltration, and tumor growth. Clin Cancer Res. 2011; 17:1001-1011. [PubMed: 21233403]

48. Gavrilyuk JI, et al. An efficient chemical approach to bispecific antibodies and antibodies of high valency. Bioorg Med Chem Lett. 2009; 19:3716-3720. [PubMed: 19497743]

49. Thomas JD, et al. Application of strain-promoted azide-alkyne cycloaddition and tetrazine ligation to targeted Fc-drug conjugates. Bioconjug Chem. 2012; 23:2007-2013. [PubMed: 22988967]

50. Wuellner U, et al. Expanding the concept of chemically programmable antibodies to RNA aptamers: chemically programmed biotherapeutics. Angew Chem Int Ed Engl. 2010; 49:59345937. [PubMed: 20645365]

51. Kontermann R. Dual targeting strategies with bispecific antibodies. mAbs. 2012; 4:182-197.

52. Doppalapudi VR, et al. Chemical generation of bispecific antibodies. Proc Natl Acad Sci U S A. 2010; 107:22611-22616. [PubMed: 21149738]

53. Rader C. DARTs take aim at BiTEs. Blood. 2011; 117:4403-4404. [PubMed: 21527536]

54. Frankel SR, Baeuerle PA. Targeting T cells to tumor cells using bispecific antibodies. Curr Opin Chem Biol. 2013; 17:385-392. [PubMed: 23623807]

55. Shokat KM, Schultz PG. Redirecting the immune response: ligand-mediated immunogenicity. J. Am. Chem. Soc. 1991; 113:1861-1862.

56. McEnaney PJ, et al. Antibody-recruiting molecules: an emerging paradigm for engaging immune function in treating human disease. ACS Chem Biol. 2012; 7:1139-1151. [PubMed: 22758917]

57. Amato RJ, et al. A phase I study of folate immune therapy (EC90 vaccine administered with GPI-0100 adjuvant followed by EC17) in patients with renal cell carcinoma. J Immunother. 2013; 36:268-275. [PubMed: 23603861] 
58. Perdih A, Dolenc MS. Small molecule antagonists of integrin receptors. Curr Med Chem. 2010; 17:2371-2392. [PubMed: 20491638]

59. Miller WH, et al. Discovery of orally active nonpeptide vitronectin receptor antagonists based on a 2-benzazepine Gly-Asp mimetic. J Med Chem. 2000; 43:22-26. [PubMed: 10633035]

60. Rader C, et al. A humanized aldolase antibody for selective chemotherapy and adaptor immunotherapy. J Mol Biol. 2003; 332:889-899. [PubMed: 12972259]

61. Li LS, et al. Chemical adaptor immunotherapy: design, synthesis, and evaluation of novel integrintargeting devices. J Med Chem. 2004; 47:5630-5640. [PubMed: 15509162]

62. Popkov M, et al. Small molecule drug activity in melanoma models may be dramatically enhanced with an antibody effector. Int J Cancer. 2006; 119:1194-1207. [PubMed: 16570283]

63. Popkov M, et al. Instant immunity through chemically programmable vaccination and covalent self-assembly. Proc Natl Acad Sci U S A. 2009; 106:4378-4383. [PubMed: 19255430]

64. Gavrilyuk JI, et al. Beta-lactam-based approach for the chemical programming of aldolase antibody 38C2. Bioorg Med Chem Lett. 2009; 19:1421-1424. [PubMed: 19181522]

65. Goswami RK, et al. Multiple catalytic aldolase antibodies suitable for chemical programming. Bioorg Med Chem Lett. 2009; 19:3821-3824. [PubMed: 19428247]

66. Xiao J, Tolbert TJ. Site-specific chemical modification of a glycoprotein fragment expressed in yeast. Methods Mol Biol. 2011; 751:329-342. [PubMed: 21674341]

67. Zhao ZG, et al. Site-specific modification of a single-chain antibody using a novel glyoxylyl-based labeling reagent. Bioconjug Chem. 1999; 10:424-430. [PubMed: 10346874]

68. Casi G, et al. Site-specific traceless coupling of potent cytotoxic drugs to recombinant antibodies for pharmacodelivery. J Am Chem Soc. 2012; 134:5887-5892. [PubMed: 22394212]

69. Peng L, et al. Combinatorial chemistry identifies high-affinity peptidomimetics against alpha4beta1 integrin for in vivo tumor imaging. Nat Chem Biol. 2006; 2:381-389. [PubMed: 16767086]

70. Doppalapudi VR, et al. Chemically programmed antibodies: endothelin receptor targeting CovXBodies. Bioorg Med Chem Lett. 2007; 17:501-506. [PubMed: 17055724]

71. Roberts LR, et al. Kappa agonist CovX-Bodies. Bioorg Med Chem Lett. 2012; 22:4173-4178. [PubMed: 22583618]

72. Gavrilyuk J, et al. Potent inhibition of HIV-1 entry with a chemically programmed antibody aided by an efficient organocatalytic synthesis. Chembiochem. 2010; 11:2113-2118. [PubMed: 20845359]

73. Sato S, et al. Chemically Programmed Antibodies as HIV-1 Attachment Inhibitors. ACS Med Chem Lett. 2013; 4:460-465. [PubMed: 23750312]

74. Hayakawa M, et al. A chemically programmed antibody is a long-lasting and potent inhibitor of influenza neuraminidase. Chembiochem. 2012; 13:2191-2195. [PubMed: 22965667]

75. Coronella $\mathbf{J}$, et al. Selective activity against proliferating tumor endothelial cells by CVX-22, a thrombospondin-1 mimetic CovX-Body. Anticancer Res. 2009; 29:2243-2252. [PubMed: 19528489]

76. Li L, et al. Antitumor efficacy of a thrombospondin 1 mimetic CovX-body. Transl Oncol. 2011; 4:249-257. [PubMed: 21804921]

77. Palanki MS, et al. Development of a long acting human growth hormone analog suitable for once a week dosing. Bioorg Med Chem Lett. 2013; 23:402-406. [PubMed: 23253442]

78. Huang J, et al. Development of a novel long-acting antidiabetic FGF21 mimetic by targeted conjugation to a scaffold antibody. J Pharmacol Exp Ther. 2013; 346:270-280. [PubMed: 23720456]

79. Triana-Baltzer G, et al. Predictive markers of efficacy for an angiopoietin-2 targeting therapeutic in xenograft models. PLoS One. 2013; 8:e80132. [PubMed: 24244628]

80. Gao Y, Kodadek T. Synthesis and screening of stereochemically diverse combinatorial libraries of peptide tertiary amides. Chem Biol. 2013; 20:360-369. [PubMed: 23521794]

81. Guo F, et al. Breaking the one antibody-one target axiom. Proc Natl Acad Sci U S A. 2006; 103:11009-11014. [PubMed: 16822849] 
82. Sinha SC, et al. Preparation of integrin alpha(v)beta3-targeting Ab 38C2 constructs. Nat Protoc. 2007; 2:449-456. [PubMed: 17406606]

83. Goswami RK, et al. Chemically programmed antibodies targeting multiple alpha(v) integrins and their effects on tumor-related functions in vitro. Bioconjug Chem. 2011; 22:1535-1544. [PubMed: 21774545]

84. Goswami RK, et al. Synthesis and evaluation of the aldolase antibody-derived chemical-antibodies targeting alpha5beta1 integrin. Mol Pharm. 2013; 10:538-543. [PubMed: 23102054]

85. Thomas JD, et al. Application of a trifunctional reactive linker for the construction of antibodydrug hybrid conjugates. Bioorg Med Chem Lett. 2008; 18:5785-5788. [PubMed: 18922692] 


\section{Highlights}

- cpAbs are a new class of pharmaceuticals.

- Small molecules are equipped with the pharmacological properties of antibodies.

- The small molecule serves as navigator and the antibody serves as carrier.

- Several hundred patients have been treated with cpAbs in clinical trials. 
(A)

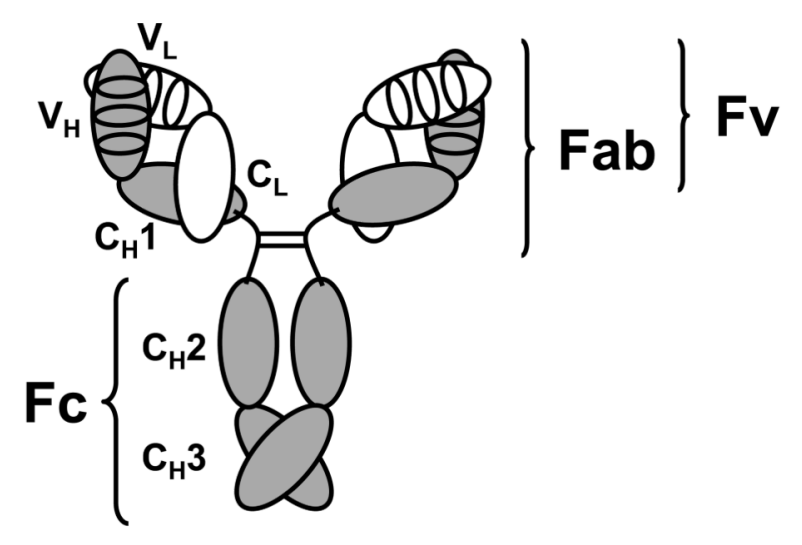

(B)
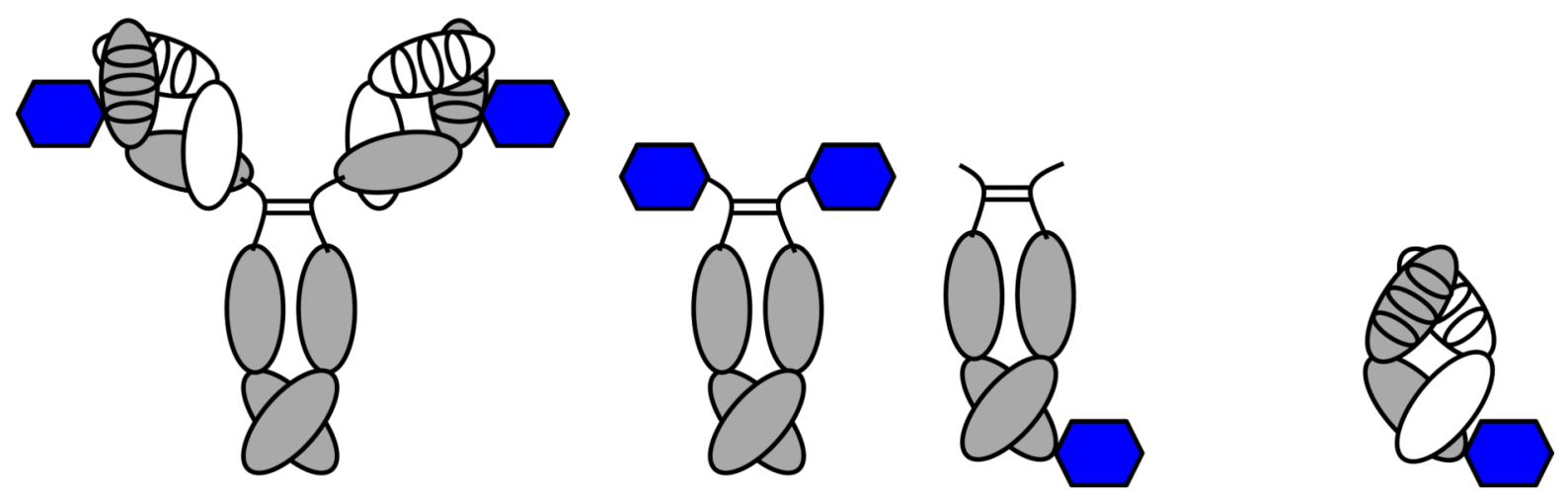

(C)
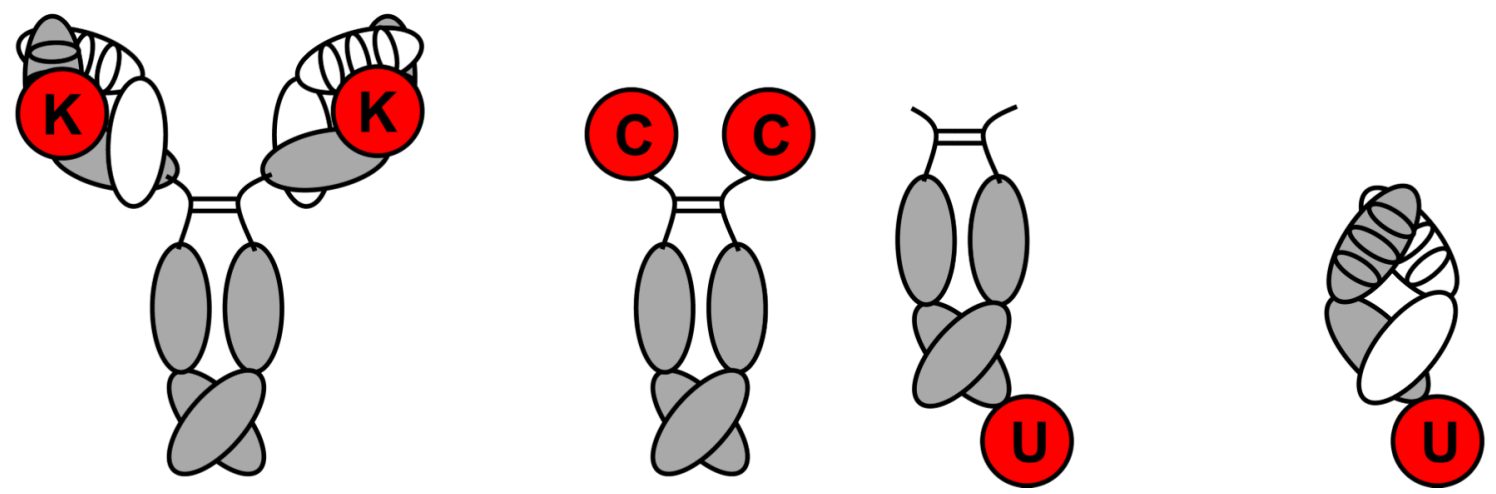

Figure 1. Comparison of the architecture of conventional mAbs and cpAbs

(A) The $150-\mathrm{kDa}$ IgG1 molecule is the dominantly used pharmaceutical format of mAbs. It contains two identical light chains (white) and two identical heavy chains (gray). The light chains consist of an $N$-terminal variable domain $\left(\mathrm{V}_{\mathrm{L}}\right)$ followed by one constant domain $\left(\mathrm{C}_{\mathrm{L}}\right)$. The heavy chains consist of an $N$-terminal variable domain $\left(\mathrm{V}_{\mathrm{H}}\right)$ followed by three constant domains $\left(\mathrm{C}_{\mathrm{H}} 1, \mathrm{C}_{\mathrm{H}} 2\right.$, and $\left.\mathrm{C}_{\mathrm{H}} 3\right) . \mathrm{C}_{\mathrm{H}} 1$ and $\mathrm{C}_{\mathrm{H}} 2$ are linked through a flexible hinge region (bent lines) that anchors four interchain disulfide bridges of the IgG1 molecule, one for each of the two light and heavy chain pairs (not shown) and two for the heavy chain pair (straight lines). The antigen binding site, or paratope, is formed by six CDRs (ovals), three provided by each variable domain. Fv, Fab, and Fc fragments of the IgG1 molecule are indicated. (B) Antigen binding in cpAbs is mediated by a synthetic component (blue hexagon) that is sitespecifically and covalently attached to an antibody component. The antibody component of cpAbs typically is a whole IgG1 molecule, which displays two synthetic components (left), or a symmetric or asymmetric Fc fragment, which displays two or one, respectively, 
synthetic components on either its $N$ - or $C$-terminus (center). In contrast to these IgG-based and Fc-based cpAbs, Fab-based cpAbs (right) bind one antigen with their synthetic component and another antigen with their antibody component. (C) Unique reactivity centers in the antibody component of cpAbs include a reactive lysine (K) residue in the paratopes of an IgG (left), engineered $N$-terminal cysteine (C) or engineered $C$-terminal selenocysteine $(\mathrm{U})$ residues of a $\mathrm{Fc}$ fragment (center), and an engineered $C$-terminal selenocysteine (U) of a Fab fragment (right). 


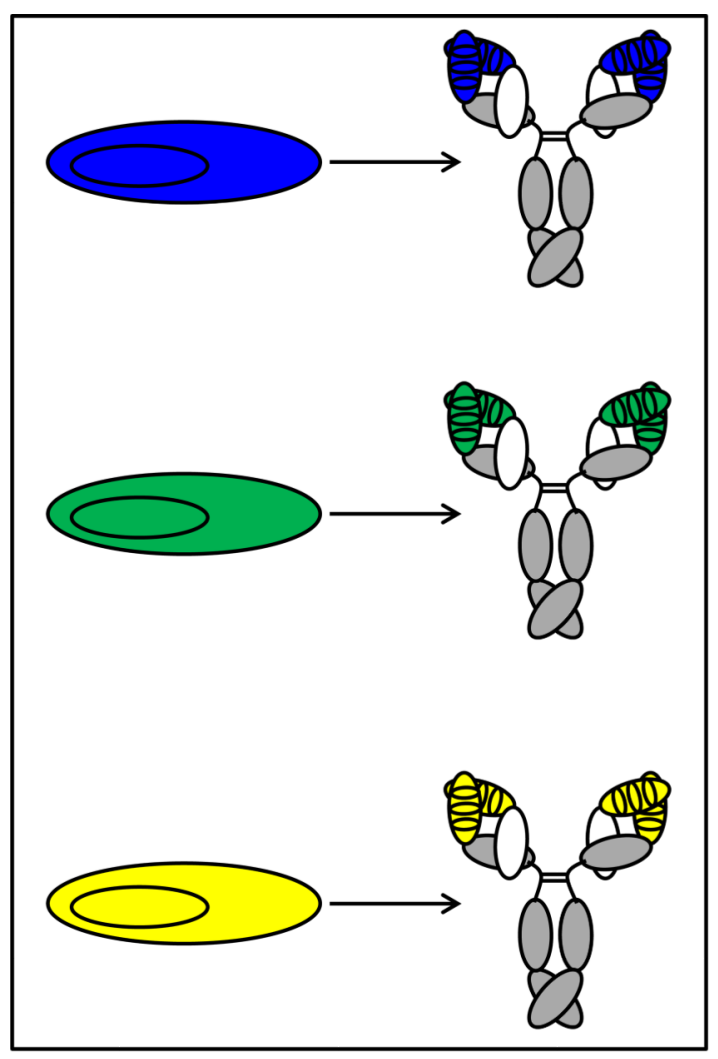

(A)

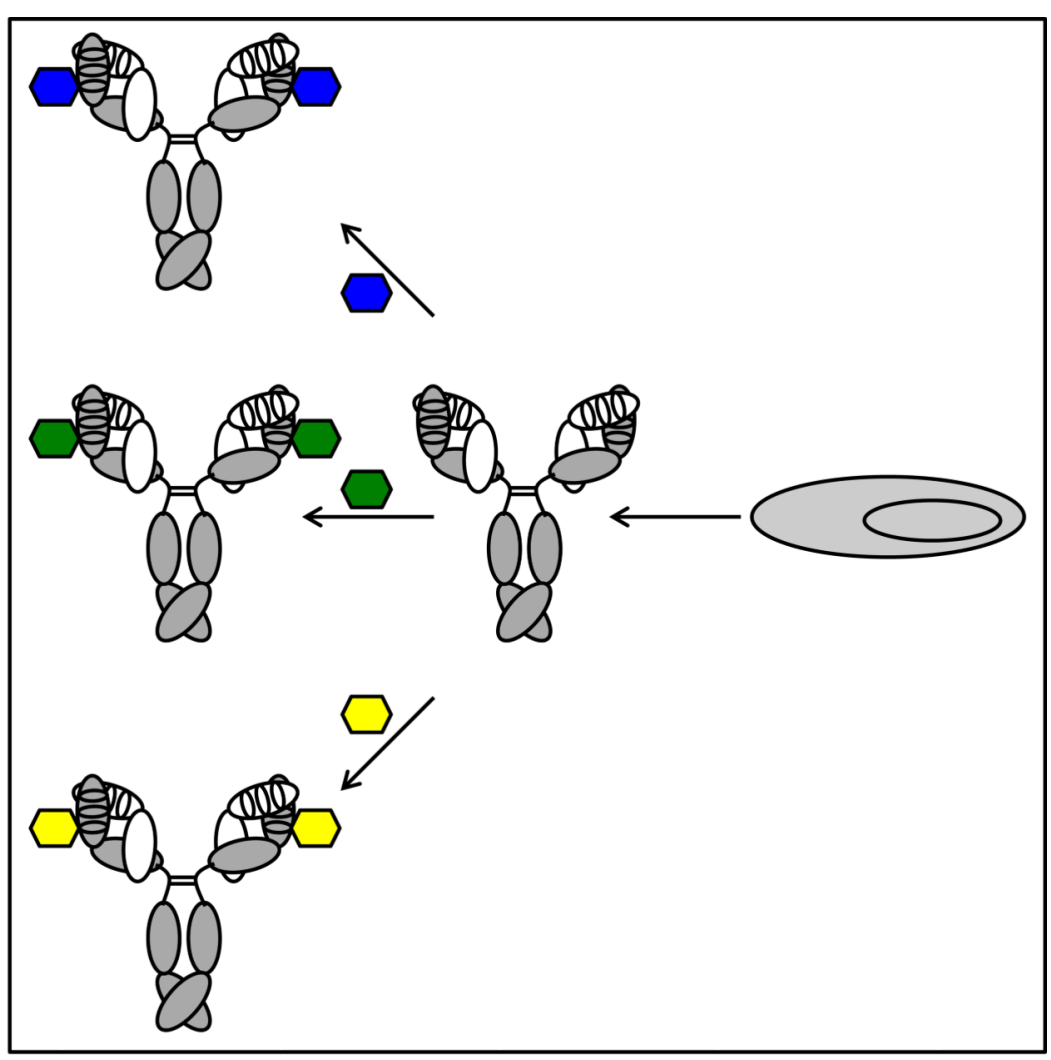

(B)

Figure 2. Comparison of the production of conventional mAbs and cpAbs

(A) In conventional $\mathrm{mAbs}$, antigen binding is mediated by the paratope, requiring the cloning, expression, and purification of different proteins for different specificities (blue, green, and yellow). (B) By contrast, cpAbs only require the manufacturing of a single protein (i.e., the antibody component), which is then chemically programmed with different small molecules (i.e., the synthetic components) for targeting different specificities. Since GMP manufacturing costs are substantially lower for small molecules compared to proteins, cpAbs are economically more attractive than conventional mAbs. Using the same protein for a range of specificities and indications also shortens drug discovery and development time. 
<smiles>[R]NC(=O)CCCC(=O)Nc1ccc(CCC(=O)CC(C)=O)cc1</smiles>

(B)<smiles>[R]CCC(=O)S[R2]</smiles><smiles>[R]CCC(=O)NC(F)CS</smiles>

(C)<smiles>[R]NC(=O)CCN1C(=O)C2=C(C)CCC21</smiles><smiles>[R]NC(=O)CCN1C(=O)CC([Se]CF)C1=O</smiles>

Figure 3. Covalent conjugation in cpAbs

Unique reactivity centers of antibody components are shown in red, synthetic components are shown in blue. (A) mAbs $38 \mathrm{C} 2$ and $\mathrm{h} 38 \mathrm{C} 2$ harbor a reactive lysine residue (red) at the base of a deep hydrophobic cleft. The nucleophilic $\varepsilon$-amino group of this reactive lysine residue can be covalently conjugated to small molecules derivatized with an electrophilic 1,3-diketone group (blue). The covalent adduct is stabilized by imine-enamine tautomerism. (B) Fc fragments with an engineered $N$-terminal cysteine display a naturally occuring 1,2aminothiol group ( $(r e d)$, which reacts with small molecules derivatized with a thioester group (blue) in the presence of sodium 2-mercaptoethanesulfonate (MESNA) by first undergoing 
transthioesterification and then $S$-to- $N$ acyl shift rearrangement under formation of an amide bond. (C) Fc fragments with an engineered $C$-terminal selenocysteine display a selenol group (red), which reacts with maleimide (blue) or iodoacetamide derivatives of small molecules. 

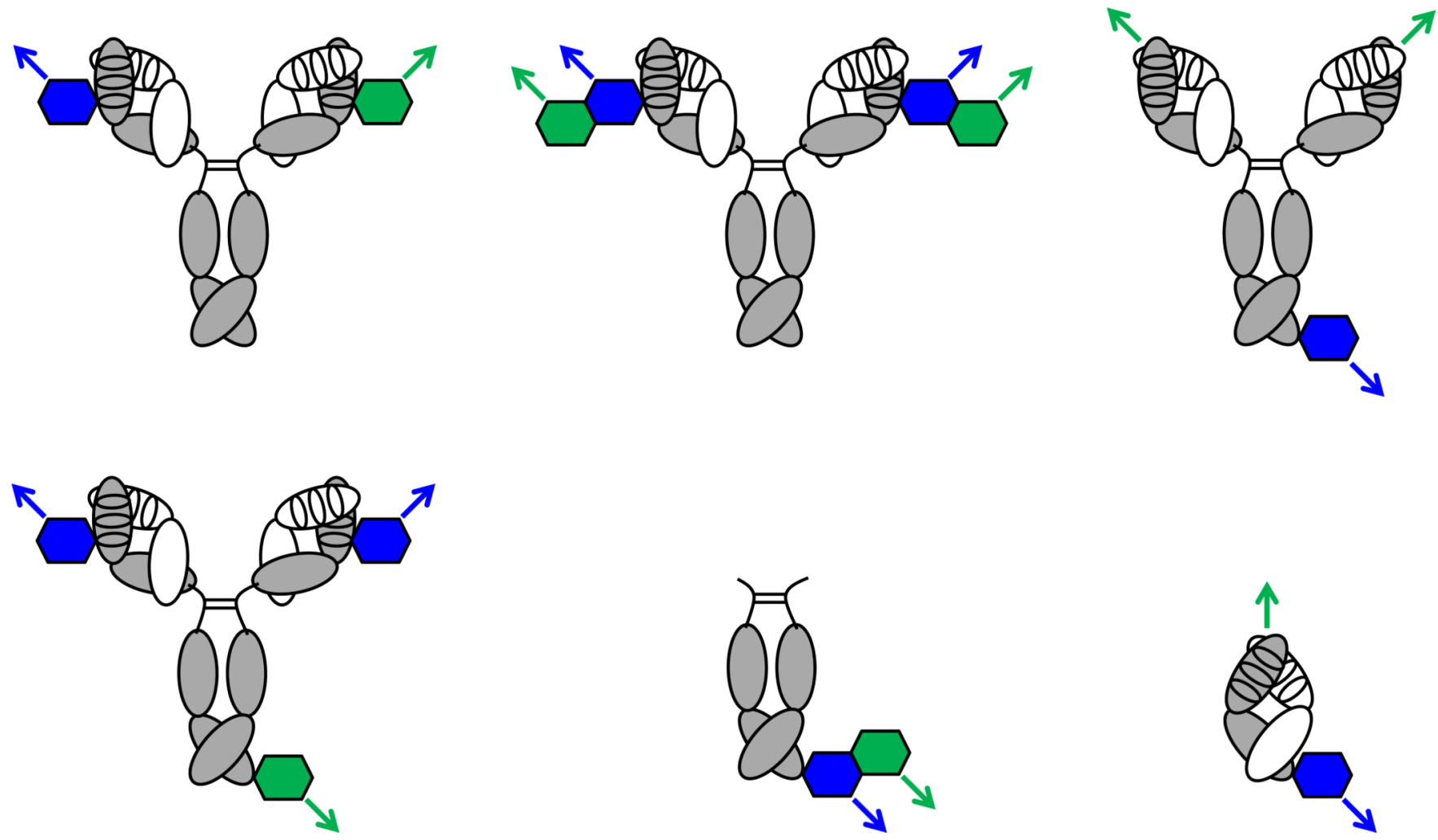

Figure 4. Bispecific cpAbs

Three conceptually different groups of bispecific cpAbs are depicted on the left, in the center, and on the right. All can simultaneously engage antigen-1 (blue arrow) and antigen-2 (green arrow). Bispecific cpAbs are generated by using two different synthetic components (blue and green hexagons) to chemically program an antibody component with two identical (top left) or two orthogonal (bottom left) unique reactivity centers. Bispecific bivalent cpAbs (top center) or bispecific monovalent cpAbs (bottom center) are generated by merging a bispecific synthetic component with an antibody component having two identical or one unique reactivity center, respectively. In cases where the antibody component of cpAbs harbors an antigen binding site that remains untouched by chemical programming, IgG-based (top right) or Fab-based (bottom right) bispecific cpAbs that bind to antigen-1 via their synthetic component and to antigen- 2 via their antibody component can be generated. 
Table 1

Comparison of small molecules, mAbs, and cpAbs.

\begin{tabular}{|l|l|l|l|}
\hline & Small molecules & mAbs & cpAbs \\
\hline Chemical diversity & unlimited & limited & unlimited \\
\hline Molecular weight & $<1 \mathrm{kDa}$ & $150 \mathrm{kDa}$ & $50-150 \mathrm{kDa}$ \\
\hline Target binding sites & 1 & 2 & $1-2$ \\
\hline Target binding specificity & medium to high & high & high \\
\hline Target binding affinity & $\mu \mathrm{M}$ to nM & $\mathrm{nM}$ & $\mathrm{nM}$ \\
\hline Target location & intracellular and extracellular & extracellular & extracellular \\
\hline Target accessibility & unlimited & limited & limited \\
\hline Circulatory half-life & minutes to hours & weeks & days to weeks \\
\hline FcRn-mediated transcytosis & no & yes & yes \\
\hline Effector functions & no & yes & yes \\
\hline Route of administration & topical, enteral, and parenteral & parenteral & parenteral \\
\hline Manufacturing costs & low & high & medium \\
\hline
\end{tabular}



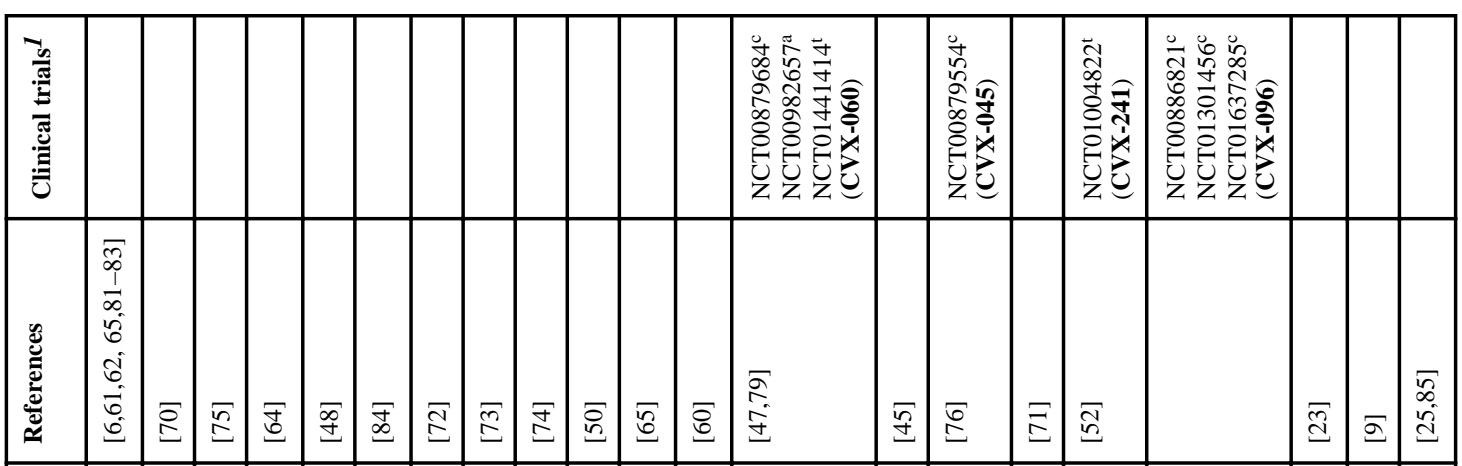

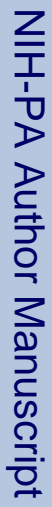
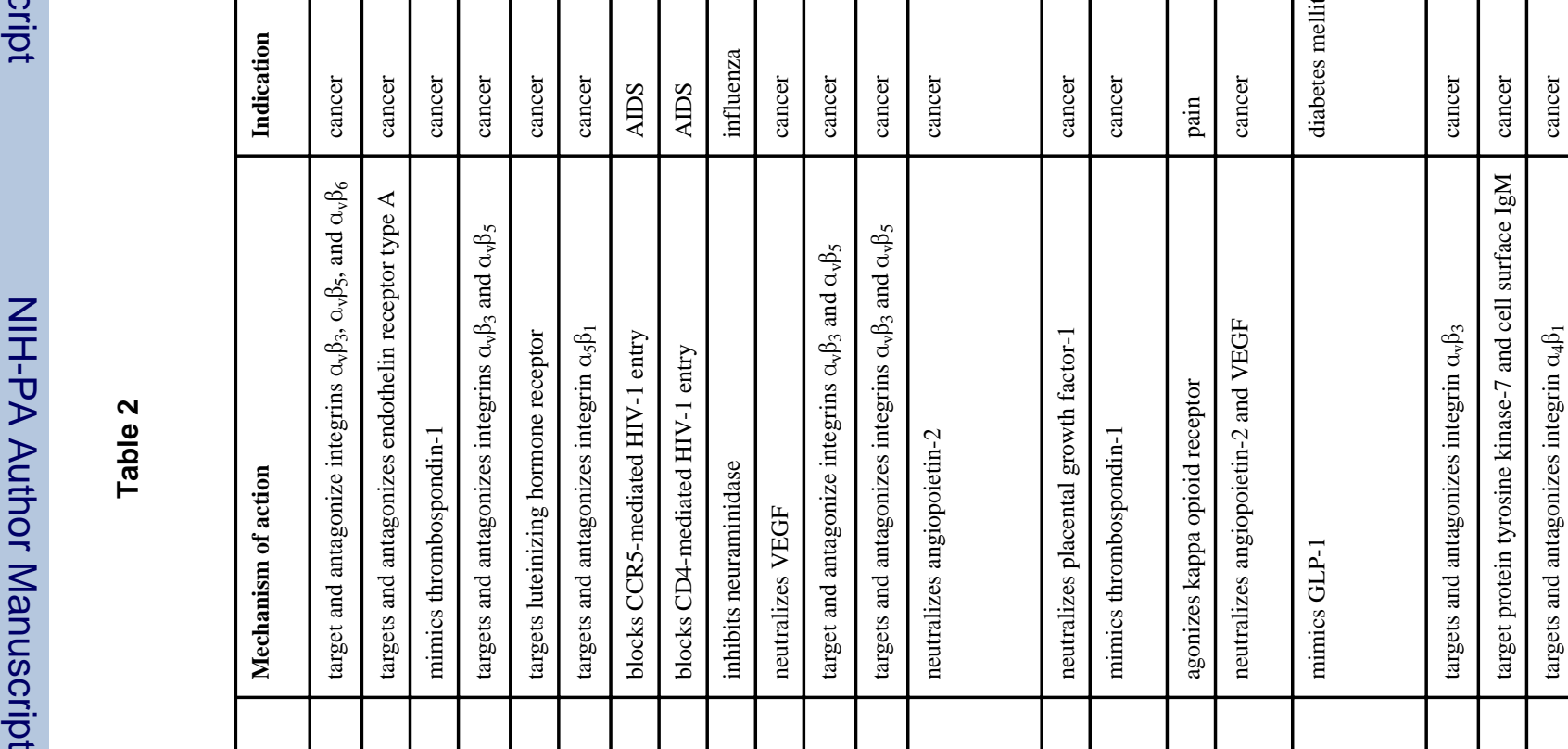

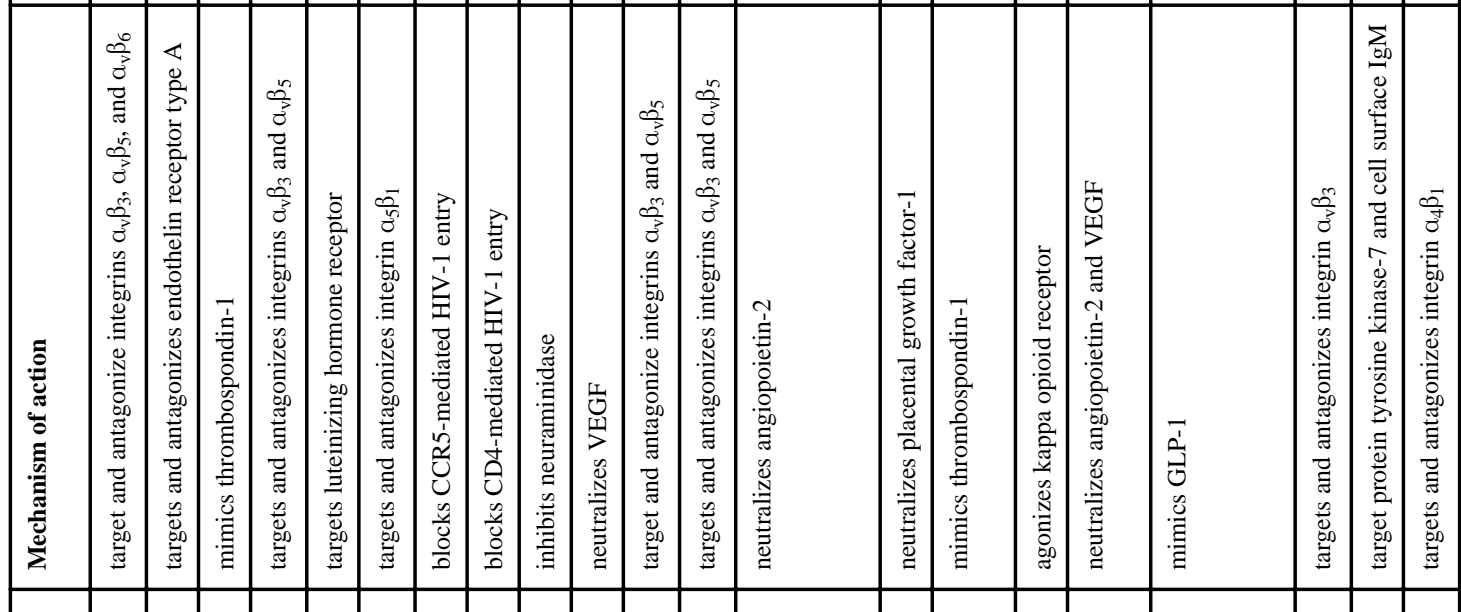

2

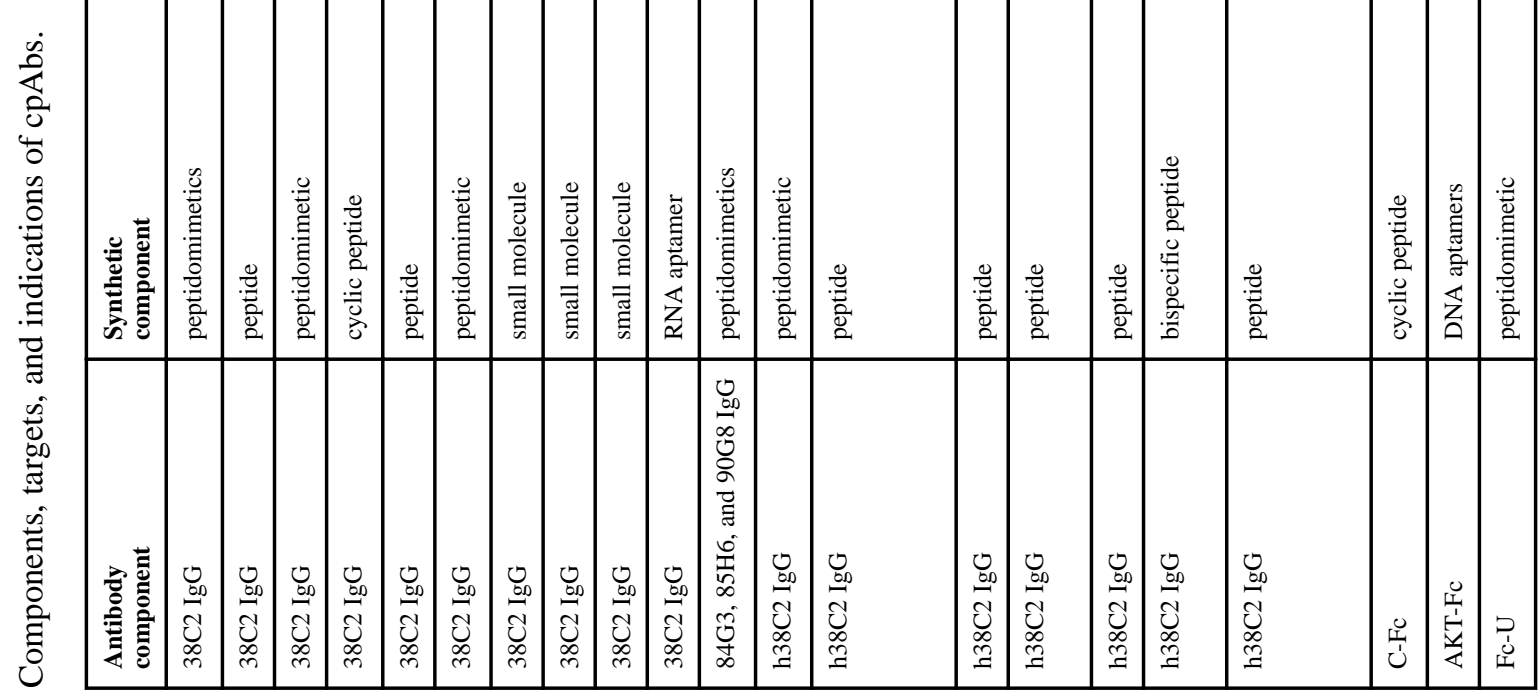




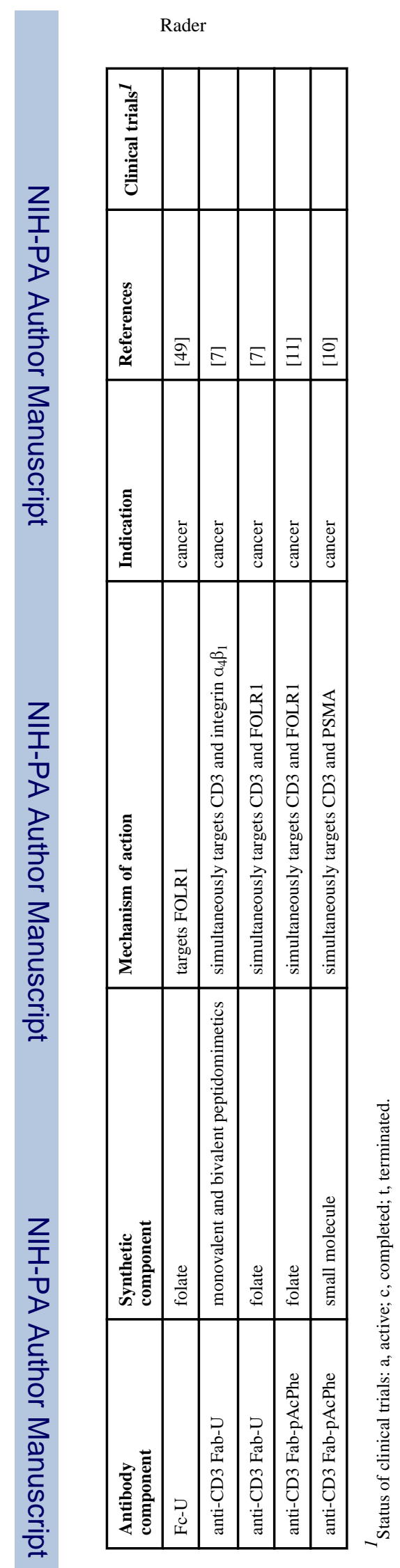

Page 25

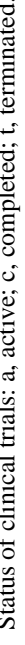

